\title{
Demonstration of an amorphous carbon tunnel diode
}

\author{
Somnath Bhattacharyya ${ }^{\text {a) }}$ and S. Ravi P. Silva \\ Nanoelectronics Centre, Advanced Technology Institute, University of Surrey, \\ Guildford GU2 7XH, United Kingdom
}

(Received 8 November 2006; accepted 9 January 2007; published online 23 February 2007)

\begin{abstract}
Negative differential conductance in metal/amorphous nitrogenated carbon $\left(a-\mathrm{CN}_{x}\right) / \mathrm{Si}$ structures is demonstrated at room temperature. These metal-insulator-semiconductor tunnel diodes are fabricated by optimizing the tunnel barrier at the $a-\mathrm{CN}_{x} / \mathrm{Si}$ junction through the control of the band gap and nitrogen doping level in carbon where this $a$-C layer acts as a semi-insulator. A small electron tunneling effective mass of about 0.06 times the free electron mass, a coherence length of $\sim 10 \mathrm{~nm}$ in these thin $a-\mathrm{CN}_{x}$ layers and a low interface trap density suggest fast device operation similar to classical tunnel diodes. (C) 2007 American Institute of Physics. [DOI: 10.1063/1.2454512]
\end{abstract}

Fabrication of tunnel junctions for fast devices has been attempted using a number of different graphitic carbon nanostructures including nanotubes, fullerenes, nanostructured amorphous carbon $(a-C)$, and nanocrystalline diamond for large area electronics. ${ }^{1-5}$ However, it is somewhat surprising that the use of amorphous diamondlike carbon (DLC) films having high breakdown voltage and a low dielectric constant together with the high thermal stability for metal/insulator/ semiconductor (MIS) diodes and MIS transistors has not been fully explored. ${ }^{5,6}$ Previously, by employing insulating DLC films or metallic graphitic films, heterojunctions have been created on crystalline silicon. ${ }^{6,7}$ However, charge accumulation at the interface or its leakage to the metal electrode without accumulation resulted due to the high defect density of states present in the films. ${ }^{8}$ Hence, direct tunneling signatures in carbon MIS structures at low bias or strong charge inversion at high bias has not been reported. ${ }^{5,9-15}$ In practice, the well-known negative differential conductance in oxide based MIS tunnel diodes or transistors has been suppressed due to the contribution of a large density of interface trap states $^{16,17}$ and limits high frequency operation. We have recently demonstrated negative differential resistance in $a-C$ quantum well structures. ${ }^{18}$ In this letter, we explore the properties of $a-\mathrm{CN}_{x} / \mathrm{Si}$ devices grown at room temperature by carefully controlling the band gap, density of states at the Fermi level $\left(E_{F}\right)$, and height of the tunnel barrier. In these $a-\mathrm{CN}_{x} / \mathrm{Si}$ heterostructures, we show features similar to those observed in state of the art direct tunnel devices with negative differential conductance. ${ }^{16}$

The samples were prepared by ablation of a high purity graphite target $(99.99 \%)$ using a $248 \mathrm{~nm}$ pulsed UV excimer laser (Lambda Physik LPX 210i). By choosing different laser fluences (4 and $16 \mathrm{~J} / \mathrm{cm}^{2}$ ) and partial pressures of nitrogen ( 5 and $100 \mathrm{mT}$ ) in the deposition chamber, films A and B were fabricated. This gives a large variation of $s p^{3}$ bonded carbon and nitrogen doping levels in the samples, which helps control the band gap $\left(E_{g}\right)$ in the range of $1-2.5 \mathrm{eV}$, respectively. These samples, up to $20 \mathrm{~nm}$ thick, were deposited at room temperature on $n$-type $\mathrm{Si}$ (111) wafers (resistivity $\sim 0.01 \Omega \mathrm{cm})$ to make $a-\mathrm{CN}_{x} / \mathrm{Si}$ heterostructures. The top contact of each device (diameter of $200 \mu \mathrm{m}$ ) was deposited by evaporating an $\sim 100 \mathrm{~nm}$ thick layer of gold.

\footnotetext{
a) Author to whom correspondence should be addressed; electronic mail:
} s.bhattacharyya@surrey.ac.uk
The diode characteristics for sample A demonstrate weak rectification behavior, which is improved by up to two orders of magnitude for sample $\mathrm{B}$, where a larger $E_{g}(\sim 2 \mathrm{eV})$ is present [Figs. 1(a) and 1(b)]. Comparing this with the low temperature characteristics we observe for sample A, the tunneling current does not show any significant change with temperature except at very low bias, close to zero (not shown here). These results are the signature for direct tunneling through a barrier created at the junction of $a-\mathrm{CN}_{x} / \mathrm{Si}$ heterostructure. This can be observed even at room temperature for sample B, with a typical signature of a negative differential
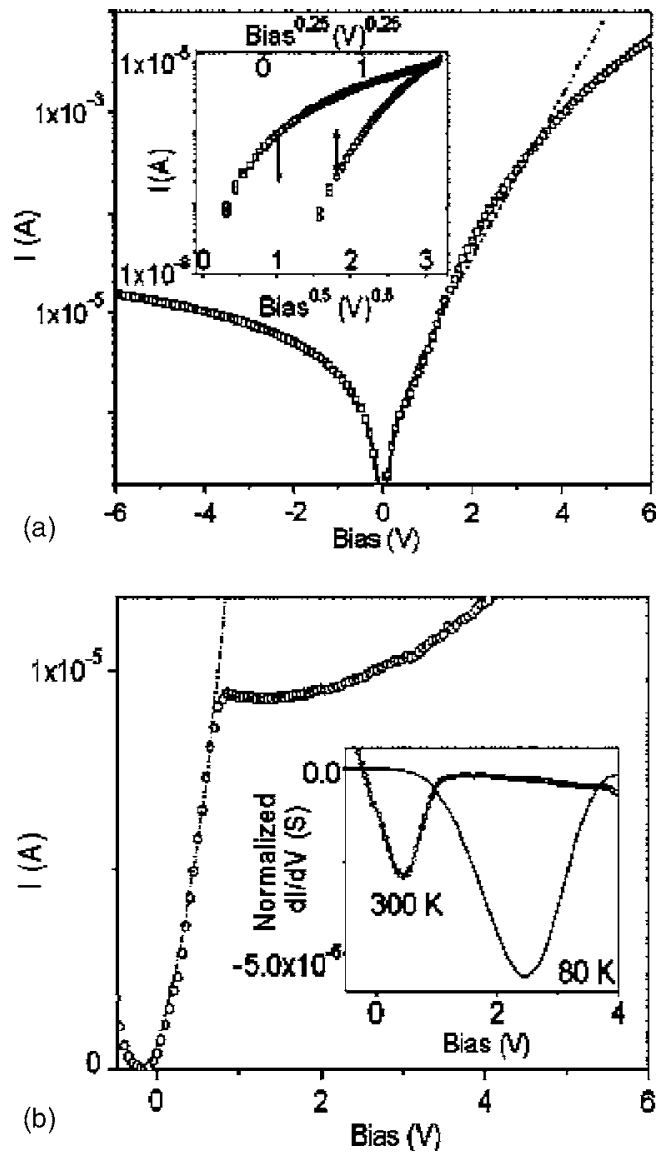

FIG. 1. (a) Room temperature $I-V$ characteristics for sample A. Inset: $I$ vs $V^{0.5}$ and $V^{0.25}$ in reverse bias. (b) Room temperature $I-V$ characteristics for sample B. Inset: Differential conductivity $(d I / d V)$ (normalized with respect to the current) vs bias. For both samples the current at low forward bias is fitted with Eq. (1). 

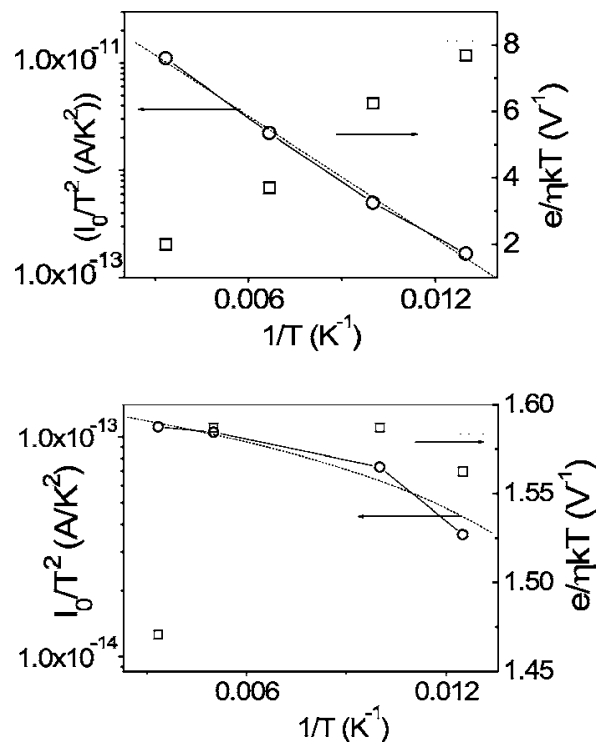

FIG. 2. $\log \left(I_{0} / T^{2}\right)$ vs $1 / T$ and ideality factor vs $1 / T$ plot in forward bias for (a) sample A and (b) sample B.

conductance [Fig. 1(b), inset], which is a major result of the current work. We explain these features, particularly for sample B, using the standard MIS tunnel diode model. ${ }^{16}$ Sample B possesses a band gap almost twice that of $n^{+}-\mathrm{Si}$ and the transfer of electrons from low $E_{g}$ (of $\mathrm{Si}$ ) to high $E_{g}$ (of $a-\mathrm{CN}_{\mathrm{x}}$ ) causes an abrupt band bending of the conduction band $\left(E_{C}\right)$ at the junction. At forward bias, the barrier to electrons gets reduced and the tunnel current shows strong nonlinear behavior (also, in sample A where $E_{g}$ is nearly equal to $\mathrm{Si}$ ). From Fig. 1(b) for sample B, we observe some depletion of charge at low bias, followed by a weak and a strong inversion at higher bias.

In reverse bias, tunneling of electron from metal to $a-\mathrm{CN}_{x} / \mathrm{Si}$ gives accumulation of charge at the junction and the conduction is governed by thermionic emission. Considering the presence of either a potential barrier or Coulomblike traps in the DLC films, this can be attributed to the Poole-Frenkel (PF) conduction, where $\log (I)$ changes linearly with $V^{1 / 2}$. . From the $V^{1 / 2}$ dependence of $\ln (I)$, in the low reverse bias region for sample $A$, we attempt to explain the data on the basis of a Schottky contact or PF conduction [Fig. 1(a), left inset]. However, an absence of linearity suggests that the variable range hopping or PF conduction is not the dominant mechanism for current conduction due to the absence of sufficient density of deep traps.

To fit the current for these devices in the low forward bias, the expression for MIS tunnel diodes based on a barrier junction (junction barrier $\phi_{b}$ and tunnel barrier $\phi_{t}$ ) as ${ }^{16}$

$$
\begin{aligned}
I= & A * T^{2} \exp \left[-2\left(\frac{2 m^{*}}{\hbar}\right)^{1 / 2}\left(q \varphi_{t}\right)^{1 / 2} d\right]\left(\exp \left\{-e \varphi_{b} / k T\right\}\right) \\
& \times\left[\exp \frac{e V}{\eta k T}-1\right]
\end{aligned}
$$

From the fit to the forward bias $I-V$ data, recorded at different temperatures, and the plot of $\ln \left(I_{0} / T^{2}\right)$ vs $1 / T$, we extract values for the $\phi_{b}$ and the diode idelity factor $(\eta)$ [Figs. 2]. The decrease of the reverse bias saturation current $\left(I_{0}\right)$ with the decrease of $-V$ could be due to lowering of $\phi_{b}$. The effective value of the Richardsons constant $A^{*}$ is found Downloaded 30 Mar 2009 to 131.227 .178 .132 . Redistribution subje to be $\sim 8 \mathrm{~A} / \mathrm{K}^{2} \mathrm{~cm}^{2}$, which points to a small value for $m^{*}$ of carbon $\left(0.06 m_{e}-0.07 m_{e}\right)$ and suggests potential applications in the form of fast tunnel devices for carbon. The tunneling probability, $T_{t} \sim \exp \left[-2\left(2 m^{*} / h^{2}\right)^{1 / 2}\left(q \phi_{t}\right)^{1 / 2} d\right]$, would increase with carrier density $\left(N_{D}\right)$ through doping (here nitrogenated $n$-type $a$-C), which also controls the band gap and the barrier heights. ${ }^{16}$ However, for $a$-C having low values of $m^{*}$ through optimizing the barrier thickness (d) of the order of $10 \mathrm{~nm}$, a value of $T_{t} \sim 10^{-10}$ can be achieved. It can even contribute to the tunneling current at room temperatures showing negative differential resistance (NDR) features. ${ }^{12}$ Also, if we consider variations of the thickness of carbon films, then the thinnest part of the films can be considered for highest $T_{t}$. Therefore, for $\phi_{b} \sim 1 \mathrm{~V}$ and $d<10 \mathrm{~nm}$, up to $10^{-6}$ of $T_{t}$ can be achieved. The value of $\phi_{b}$ varies in the range of $0.5-1 \mathrm{~V}$, which seems to be consistent with the measured band gaps of the samples and corresponds to a low density of gap states of carbon $\left(<10^{16} \mathrm{eV}^{-1} \mathrm{~cm}^{-3}\right){ }^{13}$ Also, based on the increase of $\phi_{b}$ by more than three times for sample B, compared to sample $A$, we understand that the trap density is reduced for sample $\mathrm{B}$. The activation energy calculated from $\left.\ln \left(I_{0}\right)\right)$ vs $1 / T$ plots appears to be very small $(30-60 \mathrm{meV})$, suggesting thermionic emission of carriers in the forward bias. The value of $\eta$ varies around 20 (within 18-21) for sample A and around 60 for sample B in the whole temperature range and may be considered as temperature invariant. A similar high value of $\eta$ was reported in porous silicon and also in metal/DLC/ $n$-Si/Ag structures. ${ }^{8}$ In the present structure direct tunneling through the carbon is the dominant mechanism which can explain these features. We also note that although the parameter $e / \eta \mathrm{kT}$ changes sharply at high temperatures for sample A, it saturates at low temperature $(<200 \mathrm{~K})$ for sample B, which supports the observation of direct tunneling in the present system (Fig. 2). ${ }^{5}$ From these analyses we understand that in addition to Schottky barrier emission, tunneling contributes to the total current, which depends on both $\phi_{b}$ and $\phi_{t}$ in the present devices, unlike what was previously suggested for DLC films (see Fig. 3). ${ }^{15}$ The strong nonlinearity or NDR features in $I-V$ character, which appear from the difference of $E_{g}$ at the junction of two $n$-type semiconductors and a shift of $E_{F}$ towards $E_{C}$, suggests a large deviation from the diode ideality factor due to tunneling and low density of interface states in $a-\mathrm{CN}_{x}$.

In Fig. 3 we show the energy band diagram for the $a-\mathrm{CN}_{x} / \mathrm{n}^{+} c$-Si heterojunction at zero bias. The surface potentials of silicon $\left(\psi_{c-\mathrm{Si}}\right)$ and $a-\mathrm{CN}_{x}\left(\psi_{a-\mathrm{CN}_{x}}\right)$ films are 0.18 and $0.35 \mathrm{eV}$, respectively. The work functions for these two materials, represented by $\Phi_{a-\mathrm{Si}}$ and $\Phi_{a-\mathrm{CN}_{x}}$ are 4.33 and $3 \mathrm{eV}$, respectively. Controlling tunnel barrier $\phi_{t}$ appears to be very important but this has been found challenging for $a$-C films in previous studies. ${ }^{12,15}$ The addition of nitrogen could decrease the band gap and shift $E_{F}$ from the valence band $\left(E_{V}\right)$ towards $E_{C}{ }^{10-12,19}$ As a result, $\phi_{b}$ or built in potential at the metal $/ a-\mathrm{CN}_{x}$ junction and $\phi_{t}$ at $\left(a-\mathrm{CN}_{\mathrm{x}} / \mathrm{Si}\right)$ will change in the range of $0.5-1$ and $1.5 \mathrm{eV}$, respectively. We think that due to improper choice of $d$ beyond several nanometers and $E_{g}\left(\right.$ i.e., $\phi_{t}$ ) for thick DLC films ${ }^{15}$ and for ultrathin and conducting $a$-C:H films, ${ }^{13}$ no effect of direct tunneling has been reported to date. It appears that the effect of tunneling at room temperature is small in the more conducting sample A, which acts as a conventional heterostructure or Schottky barrier junction diode or MIS tunnel diode, with a very low to AIP license or copyright; see http://apl.aip.org/apl/copyright.jsp 


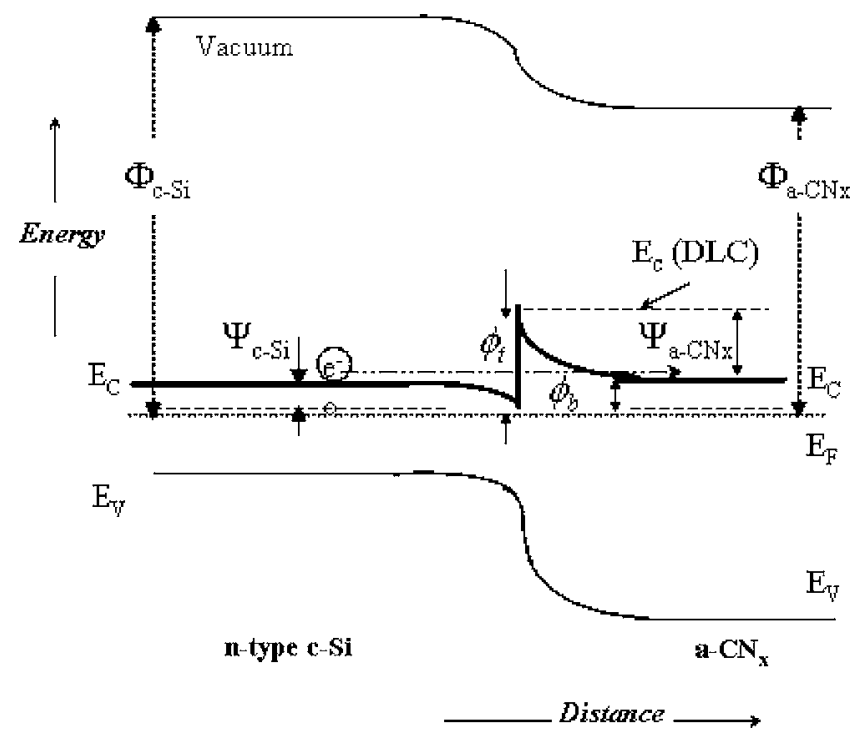

FIG. 3. Proposed energy band diagram for metal $/ a-\mathrm{CN}_{x} / n^{+} c-\mathrm{Si}$ structure at zero bias. The surface potential, work function, and energy barriers are represented by $\Phi, \psi$, and $\phi_{b}$, respectively, for $n^{+} c-\mathrm{Si}$, and $a-\mathrm{CN}_{x}$ films. The valence band, conduction band, and Fermi level for each material are denoted by $E_{V}, E_{C}$, and $E_{F}$, respectively. The donor levels and $E_{C}$ for DLC films are shown by dashed line. Electron tunneling through the barrier is represented by $e^{-}$.

value of $\phi_{b}$, typically less than $0.5 \mathrm{eV}$. This is because for sample A, the formation of a shallow donor level in the material by nitrogen incorporation is possible, which then shifts $E_{F}$ close to $E_{C}$. In Fig. 3, the structure is similar to a MIS tunnel diode with a high value of $\phi_{b}$ and $\phi_{t}$ assisting direct tunneling. Here we have presented some estimated values for $\Phi$ and $\psi$ which agree with previous reports. ${ }^{9,12,16}$

Previous studies on the photosensitivity of carbonsilicon heterojunctions showed promise for developing carbon-based optoelectronics, including photodetectors. ${ }^{20,21}$ At present the increase of current from sample A under illumination by at least two orders of magnitude is shown in Fig. 4. We think that the photogeneration of carriers takes place in the depletion region or within the $a-\mathrm{CN}_{x}$ films and photoconduction takes place in the bands separated by a large band gap. ${ }^{19,11}$ From the photocurrent of the present heterojunction we think that donor states in $a-\mathrm{CN}_{x}$ films can act as recombination centers that recombines with holes (minority carriers) in the $a-\mathrm{CN}_{x}$ films and produce an enhanced photocurrent, which could be useful in solar cell applications and photodetectors.

In summary, we show that by controlling the deposition parameters in $a-\mathrm{CN}_{x}$ films, we are able to control the effective tunnel barrier height through nitrogen doping of carbon and show heterojunction and MIS properties of these devices. We think that the observation of direct tunneling through the junction would be helpful for the fabrication of high-speed carbon based MIS tunnel diodes and other fast devices.

One of the authors (S.B.) is very much thankful to S. J. Henley and E. Mendoza for their help in experiment, J. Sh-

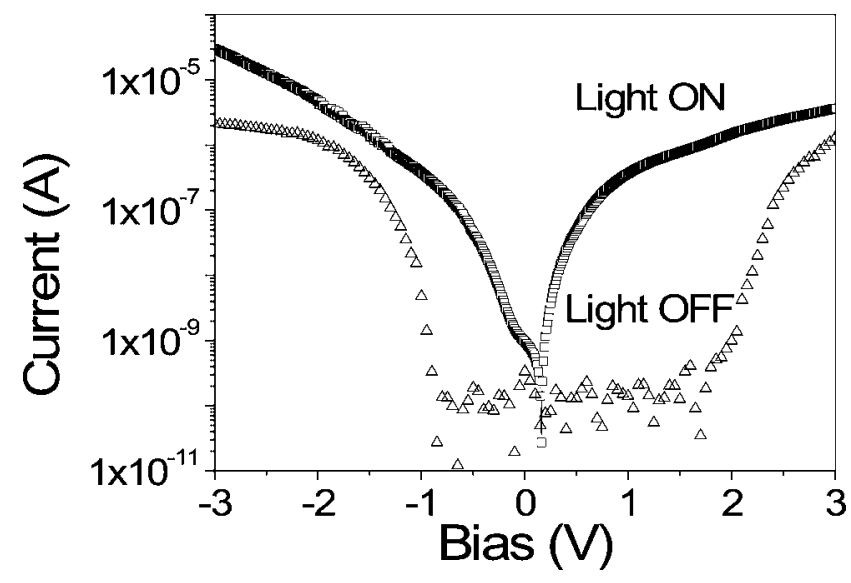

FIG. 4. $I-V$ characteristics of sample A recorded at $77 \mathrm{~K}$ in the dark and under illumination.

annon for valuable comments, and the EPSRC for financial support under the Portfolio Partnership Programmes.

${ }^{1}$ R. Martel, V. Derycke, C. Lavoie, J. Appenzeller, K. K. Chan, J. Tersoff, and Ph. Avouris, Phys. Rev. Lett. 87, 256805 (2001).

${ }^{2}$ Z. Wu, Z. Chen, X. Du, J. M. Logan, J. Sippel, M. Nikolou, K. Kamaras, J. R. Reynolds, D. B. Tanner, A. F. Hebard, and A. G. Rinzler, Science 305, 1273 (2004).

${ }^{3}$ J. O. Lee, H. Oh, J. R. Kim, K. Kang, J. J. Kim, J. Kim, and K. H. Yoo, Appl. Phys. Lett. 79, 1351 (2001).

${ }^{4}$ T. Zimmermann, M. Kubovic, A. Denisenko, K. Janischowsky, O. A. Williams, D. M. Gruen, and E. Kohn, Diamond Relat. Mater. 14, 416 (2005).

${ }^{5}$ N. A. Hastas, C. A. Dimitridis, D. H. Tassis, and S. Logothetidis, Appl. Phys. Lett. 79, 638 (2001).

${ }^{6}$ G. A. J. Amaratunga, D. A. Segal, and D. R. McKenzie, Appl. Phys. Lett. 59, 69 (1991).

${ }^{7}$ K. K. Chan, S. R. P. Silva, and G. A. J. Amaratunga, Thin Solid Films 212, 232 (1992); S. R. P. Silva and G. A. J. Amaratunga, ibid. 270, 194 (1995).

${ }^{8}$ E. Staryga and G. W. Bak, Diamond Relat. Mater. 14, 23 (2005).

${ }^{9}$ C. Ronning, U. Griesmeier, M. Gross, H. C. Hosfass, R. G. Downing, and G. P. Lamaze, Diamond Relat. Mater. 4, 666 (1995).

${ }^{10}$ N. L. Rupensinghe, R. J. Cole, M. Chhowalla, G. A. J. Amaratunga, and P. Weightman, Diamond Relat. Mater. 9, 1148 (2000).

${ }^{11}$ N. M. J. Conway, W. I. Milne, and J. Robertson, Diamond Relat. Mater. 7, 477 (1998).

${ }^{12}$ S. R. P. Silva, G. A. J. Amaratunga, C. N. Woodburn, M. E. Welland, and S. Haq, Jpn. J. Appl. Phys., Part 1 33, 6458 (1994).

${ }^{13}$ C. H. Lee and K. S. Lim, Appl. Phys. Lett. 75, 569 (1999).

${ }^{14}$ E. G. Gerstner and D. R. McKenzie, J. Appl. Phys. 84, 5647 (1998).

${ }^{15}$ S. Simeonov, A. Szekeres, E. Gyorgy, I. N. Mihaileseu, and A. Perrone, J. Appl. Phys. 95, 5111 (2004).

${ }^{16}$ S. M. Sze, Physics of Semiconductor Devices, 2nd ed. (Wiley, New York, 1981); E. M. Dons, C. S. Skowronski, and K. R. Farmer, Appl. Phys. Lett. 73, 3712 (1998).

${ }^{17}$ R. Reyes, C. Legnani, P. M. R. Pinto, M. Cremona, P. J. G. de Araujo, and C. A. Achete, Appl. Phys. Lett. 82, 4017 (2003).

${ }^{18}$ S. Bhattacharyya, S. J. Henley, E. Mendoza, L. G. Rojas, J. Allam, and S. R. P. Silva, Nat. Mater. 5, 19 (2006).

${ }^{19}$ T. Heitz, C. Godet, J. E. Bouree, B. Drevillon, and J. P. Conde, Phys. Rev. B 60, 6045 (1999).

${ }^{20}$ T. Katsuno, S. Nitta, H. Habuchi, V. Stolojan, and S. R. P. Silva, Appl. Phys. Lett. 85, 2803 (2004).

${ }^{21}$ M. Koós, I. Pócsik, and L. Tóth, Appl. Phys. Lett. 65, 2245 (1994). 